\title{
"Putting Your Money Where Your Mouth Is" - A Betting Platform for Better Prediction
}

\author{
FANG FANG* \\ College of Business Administration, California State University at San Marcos \\ MAXWELL STINCHCOMBE \\ Department of Economics, the University of Texas at Austin \\ ANDREW WHINSTON \\ Department of IROM, the University of Texas at Austin
}

\begin{abstract}
We designed a platform with a betting mechanism for eliciting costly, dispersed information of different quality. Our objective is to elicit both dispersed information and the precision of the information so as to efficiently weight dispersed information to produce reliable forecasts. After deciding to incur the costs to acquire information, participants will report their forecasts and decide the size of their bets to indicate precision. Our mechanism selects those with relatively precise information at lower costs. We also discuss the implementation issues of our mechanism and the implication to fulfil the task of crowd sourcing.
\end{abstract}

\section{Introduction}

Aggregating dispersed information can produce accurate predictions. These can be extremely useful for decision making in a world full of uncertainties. The development of the Internet provides us with a communication base to collect highly dispersed information. Therefore, how to build a reliable platform upon this communication base to centralize and integrate dispersed information becomes an academically challenging task. In this paper, we study the possibility of creating a sustainable business model by designing an efficient information elicitation and aggregation mechanism to form an accurate forecast in a cost-effective fashion.

\footnotetext{
* Contact Author. Fang Fang. College of Business Administration, California State University at San Marcos, 333 S. Twin Oaks Valley Road, San Marcos, CA 92096, U.S.A. E-mail: fangfang@csusm.edu The paper has been presented at the Workshop of Economics of E-intermediation, Paris, France, 2005 and received valuable feedback from the audience. The authors also thank Jacques Crémer, Svetlana Boyarchenko, Alfredo DiTillio, Takashi Hayashi, Dale Stahl, and Michael Whinston for many insightful comments. The authors take responsibility for all errors.
} 
When designing the reliable mechanism to produce forecasts, we not only need to elicit the dispersed information truthfully from sources but also to consider important factors such as the reliability of each piece of information and the cost to induce the truthful information. Differences in the quality of individuals' information arise from different experiences, different backgrounds, and other forms of differential access to, and competence with, information sources. Further, these differences are themselves variable across time and topics, meaning that who is better informed varies. Identifying those who have more reliable information in a specific prediction task is important for deciding how much weight to put on each person's opinion. However, each person privately holds such information and may not have an incentive to reveal it.

People who know they have more accurate information are generally willing to bet more money on it. They are willing to put their money where their mouth is. We exploit this regularity to infer the precision of people's information. Our mechanism generates reliable predictions by efficiently accuracy-weighting each person's information based on the inferred precision.

The other factor we look at is the cost for each person to acquire and share information. There is commercially useful information that is free but we study the information that people need to spend resources, money, and/or time on to acquire and assimilate. In addition, opportunity costs are another type of cost that people incur to obtain information. People will only spend and share the resources if they believe it to be worthwhile. Costs, as well as reliability, are variable and unobservable by others. For this reason, our second design task is to motivate those agents with low cost access to more reliable information to spend their resources acquiring and assimilating the information.

The early research on information elicitation mechanism design mainly focuses on eliciting a single agent's forecast of a future event (for example, Savage, 1971). More recent work on eliciting the forecasts from several agents has assumed that the quality of the forecasts is equal and there is no cost of information acquisition ${ }^{1}$ (for example, Chen et al, 2001 and 2004). Weighting observations from different sources according to their reliability has a long history in the statistics literature under the assumption that the reliability is known. Based on our knowledge, we are the first to discuss information aggregation when the reliability is only privately known. In the literature on optimal aggregation (for example, Clemen and Winkler, 1993), past performance is suggested as one possible indicator of reliability. However, such practice can only be adopted to forecasting conventional topics within a small group of pre-selected experts. In recent business applications, the range of forecasting topics has become wider, and historical data could be unavailable or unreliable. In this research, we propose to systematically weight opinions based on the amount of money the participants are willing to put on what they say.

Our idea of aggregating dispersed information from a large number of people falls into the category of utilizing "the wisdom of crowds" (Surowieski, 2004). The main idea is to rely upon the diversity and productivity of the crowd ${ }^{2}$ to resolve unconventional tasks. Specifically, Surowieski (2004) listed three types of problems to which the crowds may provide a better solution than that provided by a small group of selected experts. The

\footnotetext{
${ }^{1}$ In other words, the agents are born with the information. The only decision is whether to share.

${ }^{2}$ In Surowieski (2004), the crowd is referred to as a large group of people who are not verified to be knowledgeable to solve the entire task. However, as argued by Surowieski, the aggregate effect of the crowd can be outstanding in many of the applications.
} 
forecasting problem fits in the category of "cognition problems". The dispersion of participants can increase the dimension of problem solving and bring in more perspectives to identify the best solution. In order to insure the reliability of a random unknown crowd's inputs, we need to design proper incentive alignment mechanisms to discover the "good" crowds and motivate their contribution.

While advocating the utilization of the crowd's wisdom, our paper discusses a theoretical framework to better motivate and manage the crowds. Our forecasting problem has a specific task of predicting a future value. ${ }^{3}$ Example applications are the demand forecast of a certain product, the prediction of the number of seats in the U.S. Congress occupied by the Democratic Party after the next election, the overall orange production in the next year in California, and many more.

It is also worth noticing that our mechanism does not suggest that the experts' opinions are not important. In fact, the experts are among the crowds. They are, most of the time, the better crowd. Their analyses and opinions are valuable sources of the crowds' wisdom. However, utilizing only the selected experts is subject to risk. For example, the selection process may be biased due to the organizer's limited abilities to access all the candidates and to judge the best qualified group. In addition, the experts' opinions may still have a very small chance of being wrong, even when they are the most qualified group. Sometimes, they fail to (or are unable to) observe a few relevant factors. Or they can rely too much upon traditional ways of thinking to resolve unconventional problems. The crowd serves as a dispersed force to process and evaluate the experts' opinions and provide more perspectives. Members of the crowd can therefore correct possible biased opinions from experts, further increasing the reliability of the forecast.

There are electronic prediction markets simulating stock markets where the market prices reflect the aggregation of dispersed information. Such mechanisms also provide a method of "putting your money where your mouth is". Electronic prediction markets have recently proliferated and generated a great deal of public interest. ${ }^{4}$ Empirical research has collected evidence to prove the predictability of such markets in the field of political elections. Forsythe, et al (1992) provided empirical results on the 1988 presidential election and show that the market "worked extremely well, dominating opinion polls". Various researchers have designed many types of markets for different prediction tasks and report promising but different levels of predictability. For example, Forsythe et al (1995) reported that the market provides a very good forecast of the popular vote shares in 1993 Canadian federal election but a less accurate result in predicting the split of seats among parties. Wolfers and Zitzewitz (2003) provided a comprehensive review of the research work on electronic prediction markets and discussed explicitly the issue of different designs for different forecasting tasks. However, it is not fully understood how reliable

\footnotetext{
${ }^{3}$ There are two different types of problems in the literature on forecasting: probability forecasting and value forecasting. Probability forecasting focuses on predicting the probability of a future event, for example, Hillary Clinton has a $37.8 \%$ chance to become the first female president of the United States. In contrast, value forecasting attempts to predict the value of a random variable whose value will be realized in the future, for example, the Democratic Party will gain 51.6\% of the votes in the next congressional election.

4 Examples of actively trading markets are Iowa Electronic Markets (http://www.biz.uiowa.edu/iem), Hollywood Stock Exchange (http://www.hsx.com), Tradesports (http://www.tradesports.com), and Intrade (http://www.intrade.com).
} 
such markets are, nor what determines their reliability. ${ }^{5}$ Lewis-Beck (2005) surveyed the existing statistical models for producing election forecasts, compared the outcomes with those produced by electronic prediction markets, and argued that forecast production was a complicated field and it was still too early to determine that the electronic prediction market is a good enough mechanism.

The theoretical foundation for stock market prices providing good predictions are the Efficient Market Hypothesis (EMH; Fama, 1970) and the Rational Expectations Equilibrium (REE; Grossman and Stiglitz, 1976) frameworks. There are two related theoretical questions on how and whether the market price can efficiently aggregate private information. The first is a conceptual problem pointed out by Grossman and Stiglitz (1980). If the market price aggregated all available information, then no rational person would have an incentive to take part in the market since their private information is almost certainly less accurate than the aggregated information of everyone else. Further, if market participants shall pay a small cost to acquire their private information, then the only equilibrium is that no one will pay the cost, therefore, the market cannot reveal any of such information. That is, if the market somehow can perform its information aggregation function, it does not provide justifiable motivation for participants to collect information.

More generally, Milgrom and Stokey (1982) point out that when someone else is willing to make a bet against you, it reveals information making you less willing to bet against him. Your initial best estimate of the odds is based on your private information. Knowing that someone else, on the basis of his own private information, is willing to bet against you means that you know that his private information contradicts yours. This should decrease your willingness to bet. To resolve these conceptual problems, outside subsidies must be provided to guarantee that the agents correct incentives to trade, especially when people incur costs for acquiring information.

There is a third theoretical question, a type of "Lucas critique" (Lucas, 1976). If a prediction market becomes reliable and this reliability changes policy or politics, this may create strategic incentives to manipulate the market. If the strategic incentives are strong enough, they could offset any monetary losses incurred by the manipulators.

This paper seeks a more reliable incentive mechanism that can provide theoretically justifiable outcomes. We propose a novel betting mechanism in which a principal who needs the forecast will resort to a group of agents who may have some information about the subject. In our mechanism, the prediction is reliable in the sense that the principal can estimate the accuracy of the prediction from all the bets submitted. We conduct our analysis with a one-principal-multi-agent framework to resolve the following questions:

(1) How do we give agents incentives to truthfully reveal both their private information and the quality of their information?

(2) What do these incentives cost?

(3) How do we trade off the incentive costs and the benefits of the information we gather?

\footnotetext{
${ }^{5}$ Recent "failures" in the Iowa Electronic Markets were its prediction that John Kerry's chance of winning the 2004 Democratic Caucuses was less than $35 \%$, and its short-lasting prediction, apparently reacting to some small number of polls, that John Kerry's chance of winning the election was well over $60 \%$.
} 
In our proposed betting mechanism, the principal asks each agent to submit a report as a private forecast and place money as a bet on the report. After the uncertainty is realized, the agents can get rewards according to how close their reports are to the actual realization of the event and how much money they bet on the reports. With such a mechanism, the agents cannot simply say that they are "experts". They can only demonstrate their "expertise" by having the confidence to place bets on their reports.

We present a family of reward functions with two crucial properties. First, conditional on deciding to incur the cost to gather information, each agent's dominant strategy is to report his or her true forecast. Second, agents bet an amount that is monotonically increasing in the precision of their information. We discuss the choice of the optimal reward function in two different setups: (1) a simultaneous betting mechanism, where the principal commits to the same reward function for all the agents and agents bet without observing the activities of the other agents; (2) a sequential betting mechanism, where the principal dynamically adjusts the reward function for the next agent after observing each bet and the report submitted by the previous agents.

With both mechanisms, the agents incur the costs of information acquisition and bet only when their expected gain covers the costs. Therefore, the principal's choice of the reward function is essentially a choice of her willingness to pay for each piece of information. If the willingness to pay is high, then there could be more agents participating in the market, which will generate a more accurate prediction. The tradeoff is higher payments by the principal.

The rest of the paper is organized as follows. We present the forecasting model in a one-principal-multi-agent framework and discuss the mechanism design task in section 2. Section 3 analyzes a benchmark case where the principal can verify the reliability of the agents' information sources. We prove the existence of an optimal set of "agents" with relatively lower costs and higher precision from this analysis and discuss the property of the selection criterion. Section 4 presents a betting mechanism with a family of parameterized reward functions assuming that the agents' precision is not available. Section 5 extends the simultaneous betting mechanism to a sequential one and shows how the sequential betting can improve the cost-effectiveness for the principal. Section 6 concludes with a discussion of the implementation of a betting market and the implications of the crowd's wisdom.

\section{Model and analysis}

For decision purposes, a principal needs a forecast of a random variable to be realized in the future, say the next quarter sales of a new product. Access to information useful for making the forecast is dispersed among many people. At a cost, people can acquire and process their information. Both the quality of the information and the cost of acquiring it vary amongst the people involved. The principal's task is to motivate a set of agents with relatively lower cost to obtain more precise information and to report truthfully both their forecast and the reliability of their forecast. The principal can then use the reliability information to weight the agents' reports and form the aggregated prediction.

Throughout our modelling of the future random variable and the dispersed information, we assume normal distributions and quadratic utility functions. By contrast, we assume general distributions for the costs and precision of the information. We could replace the 
normal-quadratic formulation with more general distributions and utility functions, for example, replacing "lower variance" with "Blackwell information improvements", however, we strongly suspect that the cost of this generality would outweigh any gain. For example, we would be obliged to establish the existence and basic theoretical properties of an optimal weighting scheme for forecasts instead of using the explicit weighting scheme that arises in the normal-quadratic case. This scheme has the same basic properties that would arise in a more general setting.

\subsection{The forecasting model}

A risk neutral principal (for example, a firm) wants to forecast a future outcome, represented by a random variable $X \sim N\left(s_{0}, \tau_{0}^{-1}\right)$. The principal's payoff from generating a forecast $\hat{X}$ is $g(\hat{X}, x)=v-p(\hat{X}-x)^{2}$, where $x$ is the realization of $X, v$ is the value to the principal when the forecast is extremely precise, and $p(\hat{X}-x)^{2}$ is a quadratic penalty term for mistakes in the forecast.

To obtain a good prediction, the principal resorts to $N$ risk-neutral agents. Each agent $i \in I \triangleq\{1,2, \cdots, N\}$ can access a private independent information source at a cost $c_{i}>0 .^{6}$ If agent $i$ decides not to acquire the information, she has the same belief about the random variable, $X \sim N\left(s_{0}, \tau_{0}^{-1}\right)$, as the principal. If she does acquire information from her private source, she observes a private signal $s_{i}=x+\varepsilon_{i} \cdot{ }^{7}$ Here $\varepsilon_{i} \sim N\left(0, \tau_{i}^{-1}\right)$ is the random error of the forecast. The variance of the error $\tau_{i}^{-1}$, as well as its reciprocal $\tau_{i}$, measures ex ante the reliability of the signal $s_{i}$. The larger the value of $\tau_{i}$, the smaller the variance of $\tau_{i}^{-1}$ and the more reliable is $s_{i}$ as an indicator of the future value $x$. In the rest of the paper, we refer to $\tau_{i}$ as the precision of agent $i$ 's information for $i=1,2, \cdots, N$. We also call the reciprocal of the principal's prior variance $\tau_{0}$ the precision of the prior belief.

We assume that $\left\{X, \varepsilon_{1}, \cdots, \varepsilon_{N},\left(c_{1}, \tau_{1}\right), \cdots,\left(c_{N}, \tau_{N}\right)\right\}$ is an independent collection of random variables/vectors. Before acquiring information sources, the agents privately know their costs and precision, $\left(c_{i}, \tau_{i}\right)$, before they decide whether to acquire the signal $s_{i}$. The principal needs to select from among the agents those with low costs and high precisions in order to produce the forecast in a cost-effective fashion. Therefore, the pair $\left(c_{i}, \tau_{i}\right)$ is the

\footnotetext{
${ }^{6}$ In practice, an agent may have already obtained the information. Then we can assume that the agent's cost is 0 . Our cost assumption also includes the opportunity cost for the agent to be willing to reveal his information. An agent will acquire and share information only when the cost is compensated.

${ }^{7}$ In this paper, we use the simple form of signal $s_{i}=x+\varepsilon_{i}$. However, in the real world, the signal an agent observes may be of a more complicated form. For example, $s_{i}=\alpha x+\beta+\varepsilon_{i}$, where the coefficient $\alpha$ represents the relevance of the signal and $\beta$ represents a constant shift (or bias). However, we can transform this $s_{i}$ to $s_{i}^{\prime}=x+\varepsilon_{i}^{\prime}$. The cost term $C_{i}$ is an aggregate concept representing how much the principal should pay to induce the agents to acquire information, including both the agents' actual cost to acquire and process the signal and their opportunity cost.
} 
agents' ex ante type the principal wants to identify, which is the goal of our mechanism design.

The pairs of $\left(c_{i}, \tau_{i}\right)_{i \in I}$ are drawn from a commonly known distribution, $Q\left(\left(c_{i}, \tau_{i}\right)_{i \in I}\right)$, with $F(\tau)$ being the cumulative distribution function of the marginal distribution of the $\tau_{i}$, and $H(c)$ being the cumulative distribution function of the marginal distribution of the $c_{i}$. We assume throughout that both distributions have finite expectations.

\subsection{The optimal aggregation problem}

If a subset of agents, $S \subseteq I$, acquires their private signals and report both $s_{i}$ and $\tau_{i}$ truthfully, Lemma 1 describes the optimal way to aggregate those signals.

Lemma 1. When agents $i \in S$ truthfully report $s_{i}$ and $\tau_{i}$ to the principal, the optimal predictor that maximizes the principal's expected payoff is $\hat{X}^{*}(S)=\frac{\tau_{0} s_{0}+\sum_{i \in S} \tau_{i} s_{i}}{\tau_{0}+\sum_{i \in S} \tau_{i}}$. $\tau^{*}(S)=\tau_{0}+\sum_{i \in S} \tau_{i}$ is the prediction precision, and $v-\frac{p}{\tau^{*}(S)}$ is the principal's expected payoff. $^{8}$

Lemma 1 defines the optimal way to aggregate dispersed information, if available. Based on this result, the optimal predictor is a weighted average of all the dispersed signals. Besides each agent's signal $s_{i}$, the precision $\tau_{i}$ is important in deciding the optimal weight of $s_{i}$. In addition, the total sum of precisions $\tau^{*}(S)$ is the only parameter in the principal's ex ante valuation function. Therefore, the principal who maximizes the ex ante expected payoff is concerned about how to increase the aggregated precision.

The principal's expected gain $v-\frac{p}{\tau^{*}(S)}$ is concave in $\tau^{*}(S)$, which indicates that the principal's incentive to increase the accuracy decreases as the aggregated prediction gets more precise. The principal is less willing to compensate an agent's cost when accumulating more signals. In addition, it could be too costly for the principal to compensate everyone. A major task for the principal is to figure out, from an efficiency point of view, which people should be tapped for information and which should not.

Another aspect of the principal's problem comes from the agents' lack of incentive to report the signals and the precision truthfully. The reasons could be various. For example, a salesperson may want to send a report lower than her estimate because she knows the forecast will be used to generate her sales quota. Sometimes, people may exaggerate the precision because they want to be considered experts. Even when an agent has no other incentive to lie about the signals, he or she may still not be willing to tell the truth, which is only a weakly dominating strategy without a proper compensation scheme. This makes the report unreliable. In our setup, our agents could also pretend to obtain the information. To establish a reliable way to encourage agents to actually acquire information and report truthfully, it is important to design a reliable incentive alignment mechanism.

\footnotetext{
${ }^{8}$ See the Appendix for all the proofs of theorems in this paper.
} 


\subsection{Mechanism design procedure}

We summarize the tasks of our mechanism design problem as follows:

(1) Each agent will have the right incentive to report both $s_{i}$ and $\tau_{i}$ truthfully.

(2) The agent who does not pay the cost to collect the information will not have an incentive to claim that she did.

(3) The mechanism efficiently selects those with relatively low cost and relatively high precision.

To disentangle the tasks, we conduct our analysis in the following steps:

First, we assume that the precision of each agent's information source is verifiable. We then look for a function $c(\bullet)$ so that only those agents with $\operatorname{costs} c_{i} \leq c\left(\tau_{i}\right)$ will acquire information. Our mechanism will, for each $\tau_{i}$, have a maximal cost that the agents are willing to incur. This chooses the random set of agents who will gather information. We show that there is an optimal function, $c^{*}(\cdot)$, and examine its basic properties. Specifically, we give conditions for obtaining a non-decreasing $c^{*}$ function that has the potential to be implemented in a betting mechanism.

Second, we design a betting mechanism with a parameterized reward function that induces the agents with sufficiently low costs relative to their precision to acquire information, and to give both their true prediction and the precision. The more confident about her knowledge someone is, the more she is willing to bet on it. We give the conditions when the optimal function $c^{*}$ can be implemented so that the agents with costs lower than $c^{*}\left(\tau_{i}\right)$ will place a positive bet in the market. We provide a direct revelation mechanism where the size of an agent's bet equals the precision of her information.

Third, we show how the principal can properly weight the agents' information and apply the betting mechanism in two different settings. In the first setting, timeliness is important. The structure of rewards is announced and all those who choose to access their information do so and submit their bets and forecasts. In the second setting, some delay is acceptable and the agents are chosen one at a time. They are asked if they are willing to incur their informational costs and make a forecast and a bet. Since the reward structure can be adjusted for later agents, this can lead to a savings for the principal.

\section{The optimal set when precisions are verifiable}

In this section, we assume that the principal can verify whether agents have reported $\left(s_{i}, \tau_{i}\right)$ truthfully. The issue left to be determined is which agents should acquire information. The idea is to find an optimal reward function, $c^{*}(\tau)$, so that the agents with precision $\tau_{i}$ and $\operatorname{cost} c_{i}$ lower than $c^{*}\left(\tau_{i}\right)$ will be willing to acquire information. We call the $c^{*}(\tau)$ the recruiting function in the sense that the function determines the set of participating agents. In the following sections, we will then relax the assumption of 
verifiable $\tau_{i}$ and discuss when we can reproduce such an optimal $c^{*}(\tau)$, as well as induce agents to report their $\tau_{i}$ s truthfully.

We will restrict our search for the optimal recruiting functions to the set of nondecreasing functions. Intuitively, higher precision sources are worth more to the principal. In addition, we will look for methods to implement the optimal recruiting function in section 4 and the set of implementable recruiting functions needs to be non-decreasing. Otherwise, high precision agents will have an incentive to pretend that they are the low precision ones. Finally, under certain assumptions on the joint distribution of precision and costs discussed later, the set of non-decreasing contracts is compact.

Pick an arbitrary non-decreasing recruiting function $c(\tau)$. That is, an agent with precision $\tau_{i}$ will get paid $c\left(\tau_{i}\right)$ if she acquires the information and shares it with the principal. It can be shown that the set of agents who will collect information is $S(c)=\left\{i: 0 \leq c_{i} \leq c\left(\tau_{i}\right)\right\}$. The principal's decision is to choose an optimal reward function $c^{*}(\tau)$ from the set of non-decreasing functional forms $\mathcal{C} \triangleq\left\{c(\bullet): c\left(\tau_{1}\right) \leq c\left(\tau_{2}\right)\right.$ for $\left.\forall \tau_{1} \leq \tau_{2}\right\}$.

Let $\vec{c}=\left(c_{i}\right)_{i=1}^{N}, \vec{\tau}=\left(\tau_{i}\right)_{i=1}^{N}, \omega_{i}=\left(c_{i}, \tau_{i}\right)$, and $\vec{\omega}=\left(\omega_{i}\right)_{i=1}^{N}$. $Q$ denotes the joint distribution of $\vec{\omega} \in\left(\mathbb{R}_{+}^{2}\right)^{N}$. We use $d Q\left(\omega_{i}\right)$ to indicate integration with respect to the marginal of $Q$ on the ith component of $\left(\mathbb{R}_{+}^{2}\right)^{N}$.

Let $\Psi$ be the principal's overall payoff, which is affected by the actual group of agents the principal faces $(\vec{c}, \vec{\tau})$ and by the reward function the principal adopted to select the agents $c(\bullet)$. The payoff $\Psi(c(\bullet),(\vec{c}, \vec{\tau}))$ is then composed of two parts, the expected gain from the prediction

$$
\Psi_{1} \triangleq E\left[g\left(\hat{X}^{*}(S(c)), X\right) \mid\left(s_{i}, \tau_{i}\right)_{i \in S(c)}\right]=v-\frac{p}{\tau^{*}(S(c))}
$$

and the cost to generate the prediction $\Psi_{2} \triangleq \sum_{i \in S(c)} c\left(\tau_{i}\right)$. In the above equation of $\Psi_{1}$, $\hat{X}^{*}(S)$ and $\tau^{*}(S)$ are the optimal predictor and the overall precision of the predictor defined in Lemma 1.

Therefore, the principal's expected payoff $\Psi$ is

$$
\Psi(c(\bullet),(\vec{c}, \vec{\tau}))=\Psi_{1}-\Psi_{2}=v-\frac{p}{\tau^{*}(S(c))}-\sum_{i \in S(c)} c\left(\tau_{i}\right) .
$$

$\Psi_{1}=v-\frac{p}{\tau^{*}(S(c))}$ is increasing, concave, and takes values in the interval $\left[v-\frac{p}{\tau_{0}}, v\right)$ as $\tau^{*}(S)$ takes values in the interval $\left[\tau_{0},+\infty\right)$. The function being increasing means that receiving reports from more people increases precision. The concavity means that, on average, the return to receiving reports from more people is decreasing.

The set $S$ is uniquely determined given the cutoff $c(\tau)$. The principal's problem is to choose the optimal $c^{*}(\tau)$ so as to optimize the expected rewards, 


$$
\begin{aligned}
\Psi^{*} & =\max _{c \in \mathcal{C}} E \Psi(c(\bullet),(\vec{c}, \vec{\tau})) \\
& =v-\int_{\left(\mathbb{R}_{+}^{2}\right)^{N}} \frac{p}{\tau^{*}\left(s\left(c^{*}\right)\right)} d Q(\vec{c}, \vec{\tau})-\int_{\left(\mathbb{R}_{+}^{2}\right)^{N}} \sum_{i \in S\left(c^{*}\right)} c^{*}\left(\tau_{i}\right) d Q(\vec{c}, \vec{\tau}) .
\end{aligned}
$$

\subsection{Existence of optimal recruiting function}

An optimal recruiting function exists under certain general assumptions of the agents' distribution $Q$. For example, if there is an atomic part of $Q$, then the principal's reward function is not continuous on $c$, and an optimal curve may not be well-defined.

Assumption 1. The conditional distribution function $H(\cdot \mid \tau)$ has the property that, for any $\tau_{2}>\tau_{1} \geq 0, H\left(c \mid \tau_{1}\right) \geq H\left(c \mid \tau_{2}\right)$ for any $c>0$.

Proposition 1 (Existence). If Assumption 1 holds, then the problem $\max _{c \in \mathcal{C}} E \Psi(c(\cdot),(\vec{c}, \vec{\tau}))$ has a solution. That is, there exists an increasing optimal recruiting function $c^{*}(\tau)$.

In summary, there is no loss in restricting attention to those recruiting functions $c \in \mathcal{C}(p)$ that are bounded above by $p$ because the maximal benefit in reducing uncertainty to 0 is bounded above by $p$. Indicators of the subgraphs of such $c$ functions form an $L^{1}$ compact set and dominated convergence shows that the expected payoffs are continuous.

\subsection{A special case}

The search for an optimal curve is not an easy task. Simulation methods could be used. The optimal curve depends on both how much the principal desires an accurate forecast (that is, $p$ ) and the distribution of agents' type $Q$. It is not likely to find an analytical solution of $c^{*}(\tau)$ for a general $Q$.

One relatively easy special case has precision and costs independent, and uniformly distributed on $[0, \bar{\tau}]$ and $[0, \bar{c}]$. Equivalently, $Q$ is the uniform distribution on $([0, \bar{\tau}] \times[0, \bar{c}])^{N}$. In this case, we can study the first-order derivative of Equation (1) and show that it is uniquely satisfied by a recruiting function. The uniqueness arises from a contraction mapping and the lattice structure of the set of non-decreasing functions makes the analysis of the optimal contract transparent.

The density of each agent's precision and cost is $q\left(\tau^{\circ}, c^{\circ}\right)=\frac{1}{\bar{\tau} \bar{c}}$, the conditional cumulative distribution function of costs given precision is $H\left(c^{\circ} \mid \tau^{\circ}\right)=\frac{c^{\circ}}{\bar{c}}$, and the marginal of $i$ 's precision is $\varphi\left(\tau^{\circ}\right)=\frac{1}{\tau}$. Let $C^{*}=\left\{\left(\tau_{i}, c_{i}\right) \in \mathbb{R}_{+}^{2}: c_{i} \leq c^{*}\left(\tau_{i}\right)\right\}$ denote the subgraph of $c^{*}(\bullet)$. Taking first order derivatives on both sides of Equation (1), we obtain

$$
p \cdot \mathrm{M}(\tau)=\left[c^{*}(\tau)+\frac{c^{*}(\tau)}{\bar{c}} \frac{1 / \bar{\tau}}{1 /(\bar{\tau})}\right]=2 c^{*}(\tau)
$$

where 


$$
\mathrm{M}\left(\tau^{\circ}\right)=\int_{\left(\mathbb{R}_{+}^{2}\right)^{N-1}}\left\{\frac{1}{\tau_{0}+\sum_{j \neq i} \tau_{j} 1_{C^{*}}\left(\tau_{j}, c_{j}\right)}-\frac{1}{\tau_{0}+\tau^{\circ}+\sum_{j \neq i} \tau_{j} 1_{C^{*}}\left(\tau_{j}, c_{j}\right)}\right\} d Q_{-i}\left(\tau_{-i}, c_{-i}\right) .
$$

\section{$4 \quad$ Betting mechanism design}

In section 3 , we provided conditions where an optimal $c^{*}$ function exists and is nondecreasing. We also examined a special case when $Q$ is uniformly distributed and studied the first-order condition of $c^{*}$. In this section, we discuss when we can implement the $c^{*}$ function in a betting mechanism where we need to elicit $\left(s_{i}, \tau_{i}\right)$ for the agent $i \in S\left(c^{*}\right)$.

We now assume that the principal can neither observe nor verify an agent's signal, precision, and cost. He cannot force an agent to collect the information if the agent does not want to. Such an assumption fits the scenario of collecting the "crowd's wisdom". A proper incentive mechanism needs to be designed to elicit all the relevant information from agents. To achieve a reliable forecast, the truthful elicitation of $\left(s_{i}, \tau_{i}\right)$ has to be guaranteed. Meanwhile, the compensation should be made to those agents with sufficiently low costs relative to their precision to motivate them to acquire information.

Inspired by the folk saying, "Putting Your Money where Your Mouth Is", we design a betting mechanism that asks agents to report their information and to determine an amount of money as a bet. The agents are rewarded after the uncertainty is realized. The reward depends on how much they bet and how close their report is to the realization of the future state. The reward is designed so that an agent with the higher precision will find it optimal to place a larger bet while revealing his true forecast. This way, the agents' precision is revealed and the weights on the agents' information can then be determined.

In the rest of this section, we first show the existence of a direct revelation betting mechanism design that can induce the optimal $c^{*}(\tau)$ when it is strictly increasing, differentiable and not too concave. That is, an agent with precision $\tau_{i}$ can expect to earn $c^{*}\left(\tau_{i}\right)$. The principal can achieve the same expected payoff as if he can verify each agent's precision described in section 3 . We then discuss some potential strategies of the agents and their impact on the principal's payoff. In section 5, we extend the betting mechanism to a dynamic setting and show how the principal can improve the forecast.

\subsection{The basic betting mechanism}

To elicit $\left(s_{i}, \tau_{i}\right)$, we ask each agent to send a report $r_{i}$ as his private forecast and to place an amount of money $B_{i}$ as a bet to signal his precision. The agents are rewarded after the future state $X=x$ is realized. To provide the agents correct and quantifiable incentives to report truthfully $\left(s_{i}, \tau_{i}\right)$, the payoff they receive will depend on how close their reports are to the true realization of $x$ (that is, the agents' actual performance) and how much they bet as a signal of their expected performance. Formally, the reward is a function $f\left(B_{i}, r_{i}, x\right)$.

Given this reward structure, agent $i$ who has already acquired the signal solves

$$
\max _{r_{i}, B_{i}} E\left[\pi_{i} \mid s_{i}, \tau_{i}\right]=E\left[f\left(B_{i}, r_{i}, x\right)-B_{i} \mid s_{i}, \tau_{i}\right]
$$


for his optimal betting strategy $\left(B_{i}^{*}\left(s_{i}, \tau_{i}\right), r_{i}^{*}\left(s_{i}, \tau_{i}\right)\right)$.

Definition 1 (Revelation). We say that a betting strategy $\left(B_{i}\left(s_{i}, \tau_{i}\right), r_{i}\left(s_{i}, \tau_{i}\right)\right)$ is fully revealing if it is invertible for $\forall i \in I$. If, in addition, the strategy satisfies that $B_{i}\left(s_{i}, \tau_{i}\right)=\tau_{i}$ and $r_{i}\left(s_{i}, \tau_{i}\right)=E\left[X \mid s_{i}, \tau_{i}\right]$, we say that the strategy is directly revealing. $A$ betting mechanism that can implement the directly revealing strategy is called a direct revelation betting mechanism.

Given the betting mechanism, it occurs that an agent's expected reward is sensitive to the form of the reward function. The principal's expected payoff by implementing the betting mechanism is optimized when each agent with precision $\tau_{i}$ is rewarded $c^{*}\left(\tau_{i}\right)$ if he bets.

Definition 2. We say that a reward function $f$ implements $c^{*}(\tau)$ if $E\left[\pi_{i}\left(B_{i}^{*}, r_{i}^{*}\right) \mid s_{i}, \tau_{i}\right]=c^{*}\left(\tau_{i}\right)$ for $\forall \tau_{i} \in[0,+\infty)$.

The reward functions that implement $c^{*}$ may not be unique. In this paper, we examine the class of reward functions with the form $f\left(r_{i}, B_{i}, x\right)=g_{1}\left(B_{i}\right)-g_{2}\left(B_{i}\right)\left(r_{i}-x\right)^{2}$, where $g_{1}\left(B_{i}\right)$ and $g_{2}\left(B_{i}\right)$ are strictly positive functions. Our choice of the reward function is a modified quadratic loss function, which ensures that the agents report truthfully their private forecast of $X$ as long as $g_{2}(\cdot)$ is positive. The functions $g_{1}\left(B_{i}\right)$ and $g_{2}\left(B_{i}\right)$ need to be designed to induce the agents to bet more when they believe their precision $\tau_{i}$ is higher.

Proposition 2 (Betting function). When (1) $c^{*}(\tau)$ is non-decreasing and concave, and (2) $\left|\frac{c^{\prime \prime}(\tau)}{c^{\prime}(\tau)}\right|<2(\tau+1)^{-1}$ for $\forall \tau \in \mathbb{R}_{+}$, a reward function $f^{*}$ uniquely exists within the above class of functions that satisfies the following two conditions:

- the reward function $f^{*}$ implements $c^{*}(\tau)$;

- the agents' optimal betting strategy $\left(B_{i}^{*}, r_{i}^{*}\right)$ is directly revealing.

In addition, $f^{*}\left(r_{i}, B_{i}, x\right)=g_{1}^{*}\left(B_{i}\right)-g_{2}^{*}\left(B_{i}\right)\left(r_{i}-x\right)^{2}$, where

(1) $g_{1}^{*}\left(B_{i}\right)=c^{*}\left(B_{i}\right)+\left(B_{i}+1\right) c^{*^{\prime}}\left(B_{i}\right)+B_{i}$, and

(2) $g_{2}^{*}\left(B_{i}\right)=\left(B_{i}+1\right)^{2} c^{*^{\prime}}\left(B_{i}\right)$. 
Proposition 2 shows that the principal can achieve the same expected payoff using the betting mechanism as if he can verify the precision $\tau_{i}$ if the optimal recruiting function $c^{*}$ is "concave enough". In the special case of section 3.2, the condition $\left|\frac{c^{\prime \prime}(\tau)}{c^{\prime}(\tau)}\right|<2(\tau+1)^{-1}$ holds when $(\vec{c}, \vec{\tau})$ is uniformly distributed. Therefore, the principal can actually implement an optimal betting scheme to achieve cost-effective information elicitation and aggregation.

\subsection{Basic betting mechanism discussion}

To implement $c^{*}(\tau)$, the principal can call for a simultaneous bet to collect information from the agents. That is, a principal will announce a prediction task and a reward function as defined in Proposition 2 to let the agents bet in a short time span. Such simultaneous betting allows the principal to collect relevant information as quickly as possible because the agents, once having decided to participate, will access their information source simultaneously.

People will bet because they want to make money from their private information sources. Anonymity, as well as accountability, is important in such a betting system design. Anonymity eliminates people's reputation concern, which has been identified in literature as one potential source of reporting bias. For example, Ferderer and College (2004) reported that reputation-driven experts may herd when they are concerned that their forecasts could be bad. However, people need to be held accountable in our design because our reward function does not satisfy the limited liability condition. That is, the reward may become negative if the report differs too much from $x$, even though the overall expected reward is always non-negative (that is, $c^{*} \geq 0$ ). Therefore, the principal needs to have the ability to punish the bad bettors.

There are yet issues in such an open betting environment. Below we discuss three potential issues: multiple identities, agents' talk, and possible ex post regret.

\subsubsection{Acquisition of multiple betting identities}

In this paper, we assume that people's information is obtained from independent information sources. This assumption can be established in general if we can assume that one person only bets once. However, this is difficult when the number of agents is large and the agents are anonymous. Even though carefully designed verification processes, such as requiring one credit card per bet can restrict the chances of multiple betting, it is impossible to completely prevent.

Theorem 1 (Repeated Betting Strategy). If an agent with precision $\tau_{i}$ manages to bet twice, her expected payoff will be $2 c^{*}\left(\tau_{i}\right)$.

Theorem 1 shows that an agent can expect to double her payoffs by betting twice. Such strategic behavior has two negative impacts on the principal. First, the principal pays extra money for a piece of useless information. Second, this redundant piece of information 
affects the weights assigned to aggregate all the information, which reduces the efficiency of the final predictor. ${ }^{9}$

To reduce such a potential problem, the principal can review all the bets and weights carefully to detect potential repeated betting. Modern technologies provide some methodologies to identify issues such as repeated betting to some degree. However, this is beyond the scope of this paper.

\subsubsection{Agents' talk and signal dependency}

Our analysis assumes that agents obtain independent signals. Each agent accesses independent information sources and can contribute to the aggregate forecast with an independent point of view, improving the forecasting quality.

If two agents (for example, agent " $a$ " and "b") share their signals, the independence assumption is violated. The two have the same private forecasts and will produce the same reports and bet the same amount of money. That is:

$$
\left\{\begin{array}{c}
r_{a}=r_{b}=E\left(X \mid s_{a}, s_{b}\right) \\
B_{a}=B_{b}=\tau_{a}+\tau_{b}
\end{array}\right.
$$

This has the same effect to the principal as if one agent with precision $\tau_{a}+\tau_{b}$ bets twice, which has been discussed in section 4.2.1. Things get worse if more agents can talk together.

In addition, the principal could pay for overlapping signals if the information sources are dependent, which has a similar effect as in this case. One possible solution is for the principal to ask the agents to submit justifications for their reports and bets. If the principal observes similar justifications, it is an indication that the agents are obtaining similar signals. The principal, therefore, can adjust the weight in the aggregation to restore efficient weighting. If two reports are exactly the same, the principal can refuse the bet from one to avoid paying for the same information repeatedly.

However, verifying each agent's report significantly increases the principal's workload, especially when the potential number of bettors is large. It loses the attraction of utilizing the "crowd's wisdom", which suggests that the major computation load is accomplished by decentralized agents.

Another downside of requesting a report is the increasing cost to induce agents to bet. An agent will bet only when the expected reward covers both the cost of information acquisition $c_{i}$ and the cost of writing a report. This causes a shift in the optimal reward function $c^{*}$ and an adjustment of the reward function $f$. Essentially, it makes the principal less willing to select agents, since they are, in general, more costly. A better solution for this problem will be discussed in section 5.2.

\subsubsection{Analysis of ex-post inefficiency}

The principal picks an optimal reward function based on the prior distribution of the agent's precision and costs (that is, Q). Such a reward function is optimal in the ex ante sense. The principal's ex post payoff depends on the actual distribution of the agents'

\footnotetext{
${ }^{9}$ Following Greene (2003), we call a predictor efficient when it has the least variance among all the linear unbiased estimators. Based on this criterion, our predictor given in Lemma 1 is efficient even when we relax the normality assumptions on $X$ and $\varepsilon_{i}$ for $i=1,2, \cdots, N$.
} 
precision and costs. In this sense, the principal may regret paying too much to the agents or not getting enough information. The following two examples demonstrate two extreme cases where the principal overpaid and underpaid, respectively.

Example 1 (Overpaid). Assume that there are two agents (indexed as $i=1,2)$. Both have access to accurate information sources. That is, $\tau_{1}=\tau_{2}=\tau^{a} \rightarrow+\infty$. In addition, $\max \left\{c_{1}, c_{2}\right\}<c^{*}\left(\tau^{a}\right)$. In this case, both agents will bet and expect to be rewarded $c^{*}\left(\tau^{a}\right)$.

However, learning from either agent $i \in\{1,2\}$, the principal can obtain an accurate forecast already. The marginal benefit of learning from the other agent $j \neq i$ is $p\left(\tau_{0}+\tau^{a}\right)^{-1}-p\left(\tau_{0}+2 \tau^{a}\right)^{-1} \rightarrow 0$ while paying $c^{*}\left(\tau^{a}\right)$ to agent $j$. The principal would regret having allowed the second agent to bet because the second piece of information has no value to him.

Example 2 (Underpaid). Assume that $p=100$. The optimal recruiting curve $c^{*}(\tau)=\frac{\tau}{\tau+1}$ and the current precision $\tau^{*}=10$. The principal's expected punishment term $E\left[p(\hat{X}-X)^{2} \mid\left(s_{i}, \tau_{i}\right)_{i \in S}\right]=\frac{p}{\tau^{*}}=10$. After the forecast has been produced, the principal finds out that an agent with $\tau_{a}=6$ and cost $c_{a}=1$ did not participate because $c^{*}\left(\tau_{a}\right)=\frac{6}{7}<c_{a}=1$. If the principal incorporates this agent's information, the expected punishment term will be reduced to $\frac{p}{\tau^{*}+\tau_{a}}=6.25$, which only costs the principal $c_{a}=1$. In this sense, the principal didn't reward the agents enough. In other words, the prior belief of the agents' types, $Q$, is too optimistic.

In our specific context of information aggregation, the principal's incentive for paying for a new piece of information decreases as the current precision increases. At some point, the principal may want to stop accepting bets if he sees that the precision is accurate enough. However, he cannot do it in the simultaneous betting environment.

In section 5, we adjust the betting mechanism to a dynamic setting, where only one agent can submit a bet at a time. As we will show, such sequential betting can help reduce the problems discussed in section 4.2.

\section{$5 \quad$ Extension: Betting dynamics}

\subsection{Dynamic betting increases efficiency}

In this section, we extend the betting to a dynamic setting. The agents are arranged in a random queue to bet sequentially. For example, the principal can label the agents based on the order of their arrival. The principal allows only one agent to bet at a time. At time $t$ the principal promises agent $t$ a reward function $f_{t}\left(B_{t}, r_{t}, x\right)=g_{1}^{t}\left(B_{t}\right)-g_{2}^{t}\left(B_{t}\right)\left(r_{t}-x\right)^{2}$, where $\left(r_{t}, B_{t}\right)$ is the agent $t$ 's report and bet. The principal observes $\left(r_{t}, B_{t}\right)$ to update his belief 
on $X$. The principal will then adjust the reward function to $f_{t+1}\left(B_{t+1}, r_{t+1}, x\right)$ and allows the next agent $t+1$ to bet.

With the dynamic betting setup, the principal has the ability to adjust the reward according to the observation of how the agents' information is revealed. Therefore, the principal can observe whether the prior estimation of $Q$ is too optimistic or pessimistic. The principal can decide to stop at any time once he believes the forecast is accurate enough.

Denote $V^{t}\left(\tau^{t-1}\right)$ as the principal's expected gain from the betting in periods $t$ to $N$, which is contingent on the prediction precision the principal has collected from the previous states $\tau^{t-1}=\tau_{0}+\sum_{i=1}^{t-1} \tau_{i} \cdot 1_{c_{i} \leq c^{*}\left(\tau_{i}\right)}$, where $c^{i *}(\tau)$ is the recruiting function the principal picks at time $i$.

$$
\begin{aligned}
c^{i^{*}}\left(\tau_{i}\right) & =E\left[f_{i}\left(B_{i}^{*}, r_{i}^{*}, X\right)-B_{i}^{*} \mid s_{i}, \tau_{i}\right] \\
& =E\left[g_{1}^{i}\left(B_{i}^{*}\right)-g_{2}^{i}\left(B_{i}^{*}\right)\left(r_{i}^{*}-X\right)^{2}-B_{i}^{*} \mid s_{i}, \tau_{i}\right] \\
& =E\left[g_{1}^{i}\left(B_{i}^{*}\right)-\frac{g_{2}^{i}\left(B_{i}^{*}\right)}{\tau_{0}+\tau_{i}}-B_{i}^{*} \mid s_{i}, \tau_{i}\right]
\end{aligned}
$$

The valuation $V^{t}\left(\tau^{t-1}\right)$ is composed of two parts, the expected valuation from the next period, $\int_{\mathbb{R}_{+}^{2}} V^{t+1}\left(\tau^{t}\right) d Q\left(c_{t}, \tau_{t}\right)$, minus the expected cost to pay agent $t$ in this period. Denote $1_{c_{t} \leq c^{t}\left(\tau_{t}\right)}$ as the indicator function whether agent $t$ place a positive bet. To determine the optimal recruiting function, the principal not only needs to consider the potential contribution of the agent in the current period, but also the future rounds of betting. The problem can be modelled with the following dynamic programming formulation (6):

$$
V^{t}\left(\tau^{\mathrm{t}-1}\right)=\max _{c^{t}(\tau)} \int_{\mathbb{R}_{+}^{2}}\left[\delta V^{t+1}\left(\tau^{t-1}+\tau_{t} \cdot 1_{c_{t} \leq c^{t}\left(\tau_{t}\right)}\right)-c^{t}\left(\tau_{t}\right) \cdot 1_{c_{t} \leq c^{t}\left(\tau_{t}\right)}\right] d Q\left(c_{t}, \tau_{t}\right)
$$

where $\delta$ is the discount factor for the principal to delay the decision one more period and the terminal condition for the dynamic programming problem is

$$
V^{N+1}\left(\tau^{N}\right)=v-p \frac{1}{\tau^{N}}
$$

Define the principal's expected gain in the sequential setting as $E \Pi_{\text {seq }}^{*} \triangleq V^{1}\left(\tau^{0}=\tau_{0}\right)$ and the expected gain in the simultaneous setting as $E \Pi_{\text {simul }}^{*} \triangleq E \Psi$, as in equation (1). When $\delta=1$, the principal does not worry about making a decision early or the time interval $t$ is short. For example, the agents can gather information from sources quickly. The principal can then dynamically adjust the reward based on the ex post observation of the agents' actual types. The following proposition proves that the principal gains from the ability to dynamically adjust the reward. 
Proposition 3. When $N>1, E \Pi_{\text {seq }}^{*}(\delta=1)>E \Pi_{\text {simul }}^{*}$ always holds.

\subsection{Dynamic betting with public learning}

With dynamic betting, the principal can choose to release to the other agents what the previous agents have reported and bet. The agents can therefore learn from the previous agents' reports and update their beliefs before betting. Therefore, at time $t$, an agent $t$ 's belief of $X$ will be

$$
E\left[X \mid\left(s_{1}, \tau_{1}\right),\left(s_{2}, \tau_{2}\right), \cdots,\left(s_{t}, \tau_{t}\right)\right]=\sum_{i=1}^{t} \frac{\tau_{i}}{\tau^{t}} s_{i} 1_{c_{i} \leq c^{i \dagger}\left(\tau_{i}\right)}
$$

where $c^{i \dagger}(\tau)$ is the reward function when the agents can learn from the past. In addition, the precision of agent $t^{\prime}$ s forecast improves to $\tau^{t}$. Compared to the $c^{t^{*}}(\cdot)$ function in equation (5), the reward function $c^{i \dagger}(\tau)$ changes to:

$$
\begin{aligned}
c^{i \dagger}\left(\tau_{i}\right) & =E\left[f_{i}\left(B_{i}^{*}, r_{i}^{*}, X\right)-B_{i}^{*} \mid\left(s_{1}, \tau_{1}\right),\left(s_{2}, \tau_{2}\right), \cdots,\left(s_{i}, \tau_{i}\right)\right] \\
& =E\left[g_{1}^{i}\left(B_{i}^{*}\right)-\frac{g_{2}^{i}\left(B_{i}^{*}\right)}{\tau^{i}}-B_{i}^{*}\right] .
\end{aligned}
$$

When the principal shares previous bets and reports with agents, the principal allows the agents to observe the up-to-date information. Each agent $i$ (except for $i=1$ ) has more accurate information before she bets in such a "public learning process". The agent's reward is less risky. In addition, since the principal has the ability to adjust the reward function, she could do it in each round to exclude those agents who do not have any new piece of information to bet. Therefore, the principal can effectively eliminate the issue of repeated betting described in section 4.2.1.

In section 4.2.2, we considered the case that two agents agree to share their forecast. With such a "public learning process", the principal can effectively eliminate such incentives. This is because if two agents share their signals, only the first agent will gain from betting by submitting a more precise forecast (learned from the second agent). After the betting, both signals become available to all and the second agent will not have any new information to share in her round of betting. Therefore, the second agent's expected gain will be zero. Meanwhile, the first agent may tell the second agent what her signal is. But this signal will be shared by the principal anyway. Therefore, the second agent will make the same decision when she bets and the principal's payoff remains unaffected.

The principal can alleviate the problem of correlated signals by sharing the information with all the agents. The intuition is that the principal will share the information that has been aggregated from the past agents. The new agent will then only gain from utilizing the independent component of her signal, as shown in Proposition 4.

Proposition 4. The following two cases yield the same expected payoff to the principal: 
(3) Two agents $i$ and $j(i<j)$ have access to correlated information sources. That is, $s_{i}=x+\varepsilon_{i}$ and $s_{j}^{1}=x+\gamma \varepsilon_{i}+(1-\gamma) \varepsilon_{j}$ where $\varepsilon_{i} \sim N\left(0, \tau_{i}^{-1}\right)$ and $\varepsilon_{j} \sim N\left(0, \tau_{j}^{-1}\right)$.

(4) Two agents $i$ and $j(i<j)$ have access to independent information sources. That is, $s_{i}=x+\varepsilon_{i}$ and $s_{j}^{2}=x+\varepsilon_{j}$ where $\varepsilon_{i} \sim N\left(0, \tau_{i}^{-1}\right)$ and $\varepsilon_{j} \sim N\left(0, \tau_{j}^{-1}\right)$.

In addition, if $\gamma=1$, the agent $j$ in case (1) will not bet.

Therefore, the later agents will only seek and make money from independent information sources. This improves the cost-effectiveness of the betting mechanism by avoiding paying repeatedly for the same set of information. It also motivates agents to seek unconventional sources, which is the full utilization of the diversity of the crowds.

In the simultaneous betting setup, we discussed that one possible solution to reduce the dependency problem is for the principal to determine whether there are correlated information sources, which imposes too much burden on the principal to verify the independence if the number of agents is large. In the setting with dynamic betting and information sharing, the agents self-recommend themselves to the betting market only when they have a new independent piece of information. The principal can save significant amounts of workload by identifying the correlation among the reports submitted by the agents.

It can also be shown that the dynamic betting mechanism reduces the problem of ex post inefficiency described in section 4.2.3. Since the principal has the ability to adjust the reward function each time, she can reduce the recruiting curve to a very low level once having collected information precise enough. Therefore, the principal avoids paying too much as in Example 1. If the principal collects too little information, she can increase the reward in the next round to improve the situation as in Example 2.

Though there are significant benefits, a setting with dynamic betting and information sharing is not always strictly preferred. It comes at the cost that the principal needs to wait longer to collect all the information, especially since the agents need to observe the new recruiting curve to decide whether to incur the cost to collect the information, which sometimes could takes a long time. In addition, the principal may not want to share with the new agents the bets and reports submitted in the past rounds. This is true especially when the principal is concerned about the secrecy of the final forecast.

\section{$6 \quad$ Concluding remarks}

In this paper, we propose a theoretical framework to aggregate dispersed information. Specifically, we propose a mechanism that elicits agent's private information, as well as its quality, so as to efficiently weight the information. The implied statistical efficiency gain yields more reliable forecasts. Our mechanism provides a self-selection process where the agents can trade off between the cost to access their information and the quality of information they feel they can provide. Such a self-selection process relieves the principal's burden of evaluating the potential agents and facilitates access to a wider range of potential agents. We also discuss several variations of the market design for the principal's different prediction needs. 


\subsection{Risk neutrality and risk aversion}

Our model is the first to explicitly induce the precision of the forecast from an agent. To facilitate the demonstration, we assume that the principal and all the agents are risk neutral. If the agents are risk averse, they will bet less, and/or bet only when their precision is higher. If the agents' risk-aversion can be learnt or estimated, the principal can correspondingly adjust the way she updates the information based on the bets. However, if the agents' risk-aversion is also private information, a more complicated mechanism is required. In Chen et al (2001), a two-round market mechanism is introduced with the first round specifically designed to estimate the agents' risk-aversion. Our discussion therefore, focuses on the second round design, where the mechanism uses the agents' reports and bets to elicit their private signals and precision.

\subsection{Potential applications}

Our mechanism design for prediction production is valuable especially in the current business world where information is dispersed and it is becoming increasingly crucial to get more accurate predictions. The idea is to let independent agents self-select whether to participate based on their precision and costs. It has the potential to save the principal a large amount of time and resources to evaluate the qualification of the experts. Our betting mechanisms can be applied to many business decision scenarios.

One application is in the area of supply chain demand forecast. Guo et al (2006) discussed the importance of forecasting a macro level factor (for example, a well-defined index for a specific industry) that affects all retailers' local demand. The authors, therefore, propose to use certain market mechanisms to induce reliable forecasts of the macro factor. Our betting mechanism is a possible solution to generate such a prediction. The supply chain members can jointly organize a betting market (as the principal) and accept bets from agents all over the world as a basis for aggregating the information.

A second application can be the forecast of the sales of a new product. HewlettPackard has deployed an internal market to predict the sales of its printers (Chen and Plott, 2002). The internal market has a design similar to the stock market (also the Iowa electronic market), where participants trade shares indicating their private predictions. Their internal market utilizes market prices to convey the aggregated predictions, which are publicly observable by all the traders. The market is run internally among a small group of participants in order to avoid releasing sensitive business information outside the company. A betting market described in section 4 allows the principal to collect information from people outside the company because the principal can aggregate all the bets and reports without releasing them to the other traders. In this way, Hewlett-Packard is able to incorporate valuable information from its customers and other related sources.

In addition, the betting mechanism can also be implemented in applications such as sporting event forecasts, movie box-office forecasts, the price of a future IPO, and many others.

\subsection{Extension: Parallel methods to induce crowd's wisdom}

Our betting mechanism attempts to collect dispersed information in an ad hoc fashion. The Internet plays a crucial role in aggregating such dispersed information. As the Internet connects the world, it changes significantly how people do business. It especially facilitates unprecedented productivity in developing the projects of wikipedia, blogsphere, 
and P2P network applications such as Bit-torrent and Skype. How to better organize and utilize the power of the crowd has also stimulated a great deal of research work.

In our mechanism, we focus on the Internet's ability to bring agents together to solve a one-time unconventional problem. After the predictions are aggregated, the agents can walk away. Therefore, the principal needs to provide explicit reward functions so as to motivate the agents in this one-time interaction. Parallel research has studied long-time interactions between the principal and agents. In such a setup, a reputation mechanism may play a certain role in estimating the agents' reliability. (See Dellarocas, 2005, for discussion on reputation mechanism design and community building.) For example, the agents will exert effort to obtain the best forecast, taking account of their reputation. In addition, the principal can look at the agents' long-term performance to decide the reliability of the report. A reputation mechanism could play an important role in motivating agents to contribute to online community building and resource sharing (for example, eBay, wikipedia, and Skype). In comparison, our mechanism applies to the forecasting problems on unconventional subjects, where historical data/reputation is not available to evaluate the agents' performance.

\section{$7 \quad$ References}

Ba, S, Y. Stallaert, and A. B. Whinston (2001) "Research Commentary: Introducing a Third Dimension in Information Systems Design - The Case for Incentive Alignment," Information Systems Research, 12: 225-239.

Chen, K. Y., L. R. Fine and B. A. Huberman (2001) "Forecasting Uncertain Events with Small Groups," Proc. ACM EC’01 Conf. ACM, Tampa, FL.

Chen, K. Y., L. R. Fine and B. A. Huberman (2004) "Eliminating Public Knowledge Biases in Information-Aggregation Mechanisms," Management Science, 50: 983-994.

Chen, K. Y., and C. R. Plott (2002) "Information Aggregation Mechanisms: Concept, Design and Implementation for a Sales Forecasting Problem," CIT Social Science Working Paper.

Clemen, R., and R. Winkler (1993) "Aggregating Point Estimates: A Flexible Modelling Approach," Management Science, 39: 501 - 515.

DeGroot, M. H. (1970) Optimal Statistic Decision. McGraw-Hill: New York.

Dellarocs, C. (2005) "Reputation Mechanism Design in Online Trading Environments with Pure Moral Hazard," Information Systems Research, 16: 209-230.

Fama, E. F. (1970) "Efficient Capital Markets: A Review of Theory and Empirical Work," The Journal of Finance, 25: 383-417.

Fang, F., M. B. Stinchcombe, and A. B. Whinston (2006) "Optimal Aggregation of Information Sources with Differing Quality," CREC Working Paper, Center for Research on E-Commerce, The University of Texas at Austin. 
Ferderer, J. P., and M. College (2004) "Why Do the Blue Chip Forecasters Herd? - A Test of Information and Reputation Models," Working Paper, Macalester College.

Forsythe, R., M, Frank, V. Krishnamurthy, and T. W. Ross (1995) "Using Market Prices to Predict Election Results: The 1993 UBC Election Stock Market," The Canadian Journal of Economics, 28: 770-793.

Forsythe, R., F. Nelson, G. R. Neumann, and J. Wright (1992) "Anatomy of an Experimental Political Stock Market," American Economic Review, 82: 1142-1161.

Greene, W. H. (2003) Econometric Analysis (5th ed). Pearson Education, Inc.: Upper Saddle River, New Jersey.

Grossman, S. J. and J. Stiglitz (1976) "Information and Competitive Price Systems," American Economic Review, 66: 246-253.

Grossman, S. J. and J. Stiglitz (1980) "On the Impossibility of Informationally Efficient Markets," American Economic Review, 70: 393-408.

Guo, Z., Fang, F., and A. B. Whinston (2006) "Supply Chain Information Sharing in a Macro Prediction Market," Decision Support Systems, 42: 1944-1958.

Lewis-Beck, M. S. (2005) "Election Forecasting: Principles and Practice," The British Journal of Politics and International Relations, 7: 145-164.

Lucas, R. E. Jr. (1976) "Econometric Policy Evaluation: A Critique," Carnegie Rochester Conference Series on Public Policy, 1: 19-46.

Milgrom, P., and N. Stokey (1982) "Information, Trade, and Common Knowledge," Journal of Economic Theory, 26: 17-27.

Savage, L. J. (1971) "Elicitation of Personal Probabilities and Expectations," Journal of the American Statistical Association, 66: 783-801.

Surowiecki (2005) The Wisdom of Crowds: Why the Many Are Smarter Than the Few and How Collective Wisdom Shapes Business, Economies, Societies and Nations. Phillips \& Nelson Media, Inc.: New York.

\section{Appendix: Proofs of theorems}

Proof of Lemma 1: Given any subset $S$, If the principal observes all the agents' signals $s_{i}$ for $\forall i \in S$, the updated belief on the realization of $X$ can be expressed in the following equation (9) according to the Normal Learning Theorem (DeGroot, 1970)

$$
X \mid\left(\left(s_{i}, \tau_{i}\right)_{i \in S}\right) \sim N\left(\frac{\tau_{0} s_{0}+\sum_{i \in S} \tau_{i} s_{i}}{\tau_{0}+\sum_{i \in S} \tau_{i}}, \frac{1}{\tau_{0}+\sum_{i \in S} \tau_{i}}\right)
$$


To maximize the expected value of the prediction, the risk-neutral principal solves

$$
\begin{aligned}
& \max _{\hat{X}} E\left[g(\hat{X}, X) \mid\left(\left(s_{i}, \tau_{i}\right)_{i \in S}\right)\right] \\
&= \max _{\hat{X}}\left(v-p\left\{\hat{X}^{2}-2 \hat{X} E\left[X \mid\left(\left(s_{i}, \tau_{i}\right)_{i \in S}\right)\right]+E\left[X^{2} \mid\left(\left(s_{i}, \tau_{i}\right)_{i \in S}\right)\right]\right\}\right) \\
&= \max _{\hat{X}}\left(v-p\left\{\hat{X}-E\left[X \mid\left(\left(s_{i}, \tau_{i}\right)_{i \in S}\right)\right]\right\}^{2}-p \operatorname{var}\left[X \mid\left(\left(s_{i}, \tau_{i}\right)_{i \in S}\right)\right]\right) \\
&= \max _{\hat{X}}\left(v-p\left\{\hat{X}-\frac{\tau_{0} s_{0}+\sum_{i \in S} \tau_{i} S_{i}}{\tau_{0}+\sum_{i \in S} \tau_{i}}\right\}^{2}-\frac{p}{\tau_{0}+\sum_{i \in S} \tau_{i}}\right) \\
& \leq v-\frac{p}{\tau_{0}+\sum_{i \in S} \tau_{i}}
\end{aligned}
$$

The equality holds only when $\hat{X}^{*}(S)=\frac{\tau_{0} s_{0}+\sum_{i \in S} \tau_{i} S_{i}}{\tau_{0}+\sum_{i \in S} \tau_{i}}$. Thus, given set $S \subseteq I$, the principal's optimal predictor $\hat{X}^{*}(S)$ equals $\frac{\tau_{0} s_{0}+\sum_{i \in S} \tau_{i} S_{i}}{\tau_{0}+\sum_{i \in S} \tau_{i}}$ and expected gain is $v-\frac{p}{\tau_{0}+\sum_{i \in S} \tau_{i}}$.

Proof of Proposition 1: The full proof of this paper can be found in Fang et al (2006).

Proof of Proposition 2: Given the reward function $f\left(r_{i}, B_{i}, x\right)=g_{1}\left(B_{i}\right)-g_{2}\left(B_{i}\right)\left(r_{i}-x\right)^{2}$, agent $i$ who has obtained $s_{i}$ maximizes his utility by solving the following problem:

$$
\begin{aligned}
E \pi\left(r_{i}^{*}, B_{i}^{*}\right) & =\max _{r_{i}, B_{i}} E\left[f\left(r_{i}, B_{i}, X\right)-B_{i} \mid\left(s_{i}, \tau_{i}\right)\right] \\
& =\max _{r_{i}, B_{i}} g_{1}\left(B_{i}\right)-g_{2}\left(B_{i}\right) E\left[\left(r_{i}-X\right)^{2} \mid\left(s_{i}, \tau_{i}\right)\right]-B_{i} \\
& =\max _{r_{i}, B_{i}} g_{1}\left(B_{i}\right)-g_{2}\left(B_{i}\right)\left(r_{i}-E\left(X \mid\left(s_{i}, \tau_{i}\right)\right)\right)^{2}-\frac{g_{2}\left(B_{i}\right)}{1+\tau_{i}}-B_{i}
\end{aligned}
$$

Since $g_{2}\left(B_{i}\right)>0, r_{i}^{*}=E\left[X \mid\left(s_{i}, \tau_{i}\right)\right]$ and $B_{i}^{*}$ satisfies

$$
g_{1}^{\prime}\left(B_{i}^{*}\right)-\frac{g_{2}^{\prime}\left(B_{i}^{*}\right)}{1+\tau_{i}}-1=0
$$

which is equivalent to $\tau_{i}=\frac{g_{2}^{\prime}\left(B_{i}^{*}\right)}{g_{1}^{\prime}\left(B_{i}^{*}\right)-1}-1$.

Here, we see that agents will give their forecast of $X$ given such a quadratic reward function. If we want to implement a direct revelation mechanism, we can restrict the set of $g_{1}(\cdot)$ and $g_{2}(\cdot)$ to make $\frac{g_{2}^{\prime}\left(B_{i}^{*}\right)}{g_{1}^{\prime}\left(B_{i}^{*}\right)-1}=B_{i}+1$. The first order condition of equation (11) becomes

$$
\left(g_{1}^{\prime}\left(B_{i}^{*}\right)-1\right)\left(1-\frac{g_{2}^{\prime}\left(B_{i}^{*}\right)}{g_{1}^{\prime}\left(B_{i}^{*}\right)-1} \frac{1}{1+\tau_{i}}\right)=\left(g_{1}^{\prime}\left(B_{i}^{*}\right)-1\right)\left(1-\left(B_{i}^{*}+1\right) \frac{1}{1+\tau_{i}}\right)=0
$$


Hence, we obtain that $B_{i}^{*}=\tau_{i}$. Given our condition, we have

$$
g_{2}^{\prime}\left(B_{i}\right)=\left(B_{i}+1\right)\left(g_{2}^{\prime}\left(B_{i}\right)-1\right)=\left(B_{i} g_{1}\left(B_{i}\right)\right)^{\prime}-g_{1}\left(B_{i}\right)+g_{1}^{\prime}\left(B_{i}\right)-B_{i}-1
$$

Integrating both sides, we have

$$
g_{2}\left(B_{i}\right)=B_{i} g_{1}\left(B_{i}\right)-\int_{0}^{B_{i}} g_{1}(s) d s+g_{1}\left(B_{i}\right)-\frac{1}{2} B_{i}^{2}-B_{i}+\text { const }_{1}
$$

where "const 1 " is a constant term from integration.

Hence the expected gain of an agent is calculated as

$$
\begin{aligned}
E \pi_{i}\left(r_{i}^{*}, B_{i}^{*}\right) & =g_{1}\left(B_{i}^{*}\right)-g_{2}\left(B_{i}^{*}\right) \frac{1}{B_{i}^{*}+1}-B_{i}^{*} \\
& =g_{1}\left(B_{i}^{*}\right)-\frac{B_{i}^{*} g_{1}\left(B_{i}^{*}\right)-\int_{0}^{B_{i}^{*}} g_{1}(s) d s+g_{1}\left(B_{i}^{*}\right)-\frac{1}{2} B_{i}^{* 2}-B_{i}^{*}+\text { const }_{1}}{B_{i}^{*}+1}-B_{i}^{*} \\
& =-\frac{1}{2} B_{i}^{*}+\frac{1}{2}+\frac{\int_{0}^{B_{i}^{*}} g_{1}(s) d s-\left(\text { const }_{1}+\frac{1}{2}\right)}{B_{i}^{*}+1}
\end{aligned}
$$

In order to implement $c^{*}$, we set the right hand side of equation (13) to equal to $c^{*}$. Such an equation can be solved as follows:

$$
\begin{gathered}
\int_{0}^{B_{i}^{*}} g_{1}(s) d s=\left(B_{i}^{*}+1\right) c^{*}\left(B_{i}^{*}\right)+\frac{1}{2} B_{i}^{* 2}+\text { const }_{1} \\
\Rightarrow g_{1}\left(B_{i}^{*}\right)=c^{*}\left(B_{i}^{*}\right)+\left(B_{i}^{*}+1\right) c^{*^{\prime}}\left(B_{i}^{*}\right)+B_{i}^{*}
\end{gathered}
$$

In the above, we find $g_{1}^{*}$ and $g_{2}^{*}$ from the FOC (11) with assumption that the second order condition holds. We now check the concavity condition. It can be checked that our requirement of $g_{1}^{\prime}\left(B_{i}\right)>1$ can only be satisfied when $2 c^{*^{\prime}}\left(B_{i}^{*}\right)+\left(B_{i}^{*}+1\right) c^{*^{\prime \prime}}\left(B_{i}^{*}\right)>0$, which requires $g_{2}\left(B_{i}^{*}\right)=\left(B_{i}^{*}+1\right)^{2} c^{*^{\prime}}\left(B_{i}^{*}\right)$ to be an increasing function. Plug back in equation (12):

$$
\begin{aligned}
g_{2}\left(B_{i}^{*}\right)= & \left(B_{i}^{*}+1\right)\left(c^{*}\left(B_{i}^{*}\right)+\left(B_{i}^{*}+1\right) c^{*^{\prime}}\left(B_{i}^{*}\right)+B_{i}\right)-\frac{1}{2} B_{i}^{* 2}-B_{i}^{*}+\text { const }_{1} \\
& -\left[\left(B_{i}^{*}+1\right) c^{*}\left(B_{i}^{*}\right)+\frac{1}{2} B_{i}^{* 2}+\text { const }_{1}\right] \\
& =\left(B_{i}^{*}+1\right) c^{*^{\prime}}\left(B_{i}^{*}\right)
\end{aligned}
$$


So the constant term does not matter. Above equations (14) and (12) guarantees satisfaction of the first order condition. For the second order condition, we should have

$$
g_{1}^{\prime \prime}\left(B_{i}\right)-\frac{g_{2}^{\prime \prime}\left(B_{i}\right)}{1+\tau_{i}}<0
$$

which yields condition $-\frac{c^{\prime \prime}(\tau)}{1+\tau}<2 c^{\prime}(\tau)$, or equivalently, $\left|\frac{c^{\prime \prime}(\tau)}{c^{\prime}(\tau)}\right|<2(\tau+1)^{-1}$ since $c^{*}$ is increasing and concave.

Proof of Theorem 1: If the agent has a second chance of betting, he will solve the optimization problem in equation (4) again, which yields the same strategy. Therefore, the agent doubles her expected reward in the second betting.

Proof of Proposition 3: Assume that $\delta=1$. In sequential betting, one alternative of the principal is to choose a reward function $f$ in all $N$ rounds of betting, where $f$ is the one that implements $C^{*}$ as defined in section4. Then the principal expects the same payoff as from the simultaneous betting market. Thus, we have

$$
E\left[\Pi_{\text {seq }} \mid c^{1^{*}}=c^{2^{*}}=\cdots=c^{N^{*}}=c^{*}\right]=E \Pi_{\text {simul }} .
$$

When $N>1$, the principal's optimal valuation function in a sequential betting market before the last round is

$$
V^{N}\left(\tau^{N-1}\right)=\max _{c^{N^{*}}}\left(v-p \frac{1}{\tau^{N-1}+\tau_{N} \cdot 1_{c_{N} \leq c^{N^{*}}\left(\tau_{N}\right)}}-c^{N^{*}}\left(\tau_{N} \cdot 1_{c_{N} \leq c^{N^{*}}\left(\tau_{N}\right)}\right)\right) .
$$

It is, therefore, obvious that $c^{N^{*}}$ is variable of $\tau^{N-1}$. That is, $c^{*} \neq c^{N^{*}}$ cannot be an optimal solution to the above problem. Thus, we have: $E \Pi_{s e q}^{*}>E\left[\Pi_{s e q} \mid c^{1^{*}}=c^{2^{*}}=\cdots=c^{N^{*}}=c^{*}\right]$. Therefore, we obtain $E \Pi_{\text {seq }}^{*}>E \Pi_{\text {simul }}$

Proof of Proposition 4: In the first case, agent $j$ 's posterior belief of $X$ is

$$
X \mid\left(\left(s_{k}, \tau_{k}\right)_{k<j},\left(s_{j}^{1}, \tau_{j}^{1}\right)\right) \sim N\left(\frac{\tau^{k} s^{k}+\frac{\tau_{i}-\gamma^{2} \tau_{j}^{1}}{(1-\gamma)^{2} \tau_{i} \tau_{j}^{1}} \cdot \frac{s_{j}^{1}-\gamma s_{i}}{1-\gamma}}{\tau^{k}+\frac{\tau_{i}-\gamma^{2} \tau_{j}^{1}}{(1-\gamma)^{2} \tau_{i} \tau_{j}^{1}}}, \frac{1}{\tau^{k}+\frac{\tau_{i}-\gamma^{2} \tau_{j}^{1}}{(1-\gamma)^{2} \tau_{i} \tau_{j}^{1}}}\right),
$$

where $\tau_{j}^{1}=\frac{\tau_{i} \tau_{j}}{\gamma^{2} \tau_{j}+(1-\gamma)^{2} \tau_{i}}$ is the precision of $s_{j}^{1}$. In addition, $s^{k}$ and $\tau^{k}$ is the principal's aggregate forecast and precision before agent $j$ bets.

Since $s_{j}^{1}=x+\gamma \varepsilon_{i}+(1-\gamma) \varepsilon_{j}$, we have and $\frac{s_{j}^{1}-\gamma s_{i}}{1-\gamma}=s_{j}^{2}$ and $\frac{(1-\gamma)^{2} \tau_{i} \tau_{j}^{1}}{\tau_{i}-\gamma^{2} \tau_{j}^{1}}=\tau_{j}$. Therefore, we have, for the first case, the following result: 


$$
X \mid\left(\left(s_{k}, \tau_{k}\right)_{k<j},\left(s_{j}^{1}, \tau_{j}^{1}\right)\right) \sim N\left(\frac{\tau^{k} s^{k}+\tau_{j} s_{j}^{2}}{\tau^{k}+\tau_{j}}, \frac{1}{\tau^{k}+\tau_{j}}\right) .
$$

Meanwhile, for the second case, we can obtain the same outcome after agent $j$ ' bet:

$$
X \mid\left(\left(s_{k}, \tau_{k}\right)_{k<j},\left(s_{j}^{2}, \tau_{j}\right)\right) \sim N\left(\frac{\tau^{k} s^{k}+\tau_{j} s_{j}^{2}}{\tau^{k}+\tau_{j}}, \frac{1}{\tau^{k}+\tau_{j}}\right) .
$$

That is, these two cases yield the same outcome. 